\title{
Influence du bouchon vaseux sur les variations des concentrations en oxygène dissous Cas de l'estuaire de la Loire
}

\author{
L.A. Romana $\left({ }^{*}\right)$, B. Thouvenin $(*)$, B. Galenne $(* *)$, C. Casanova $(* *)$
}

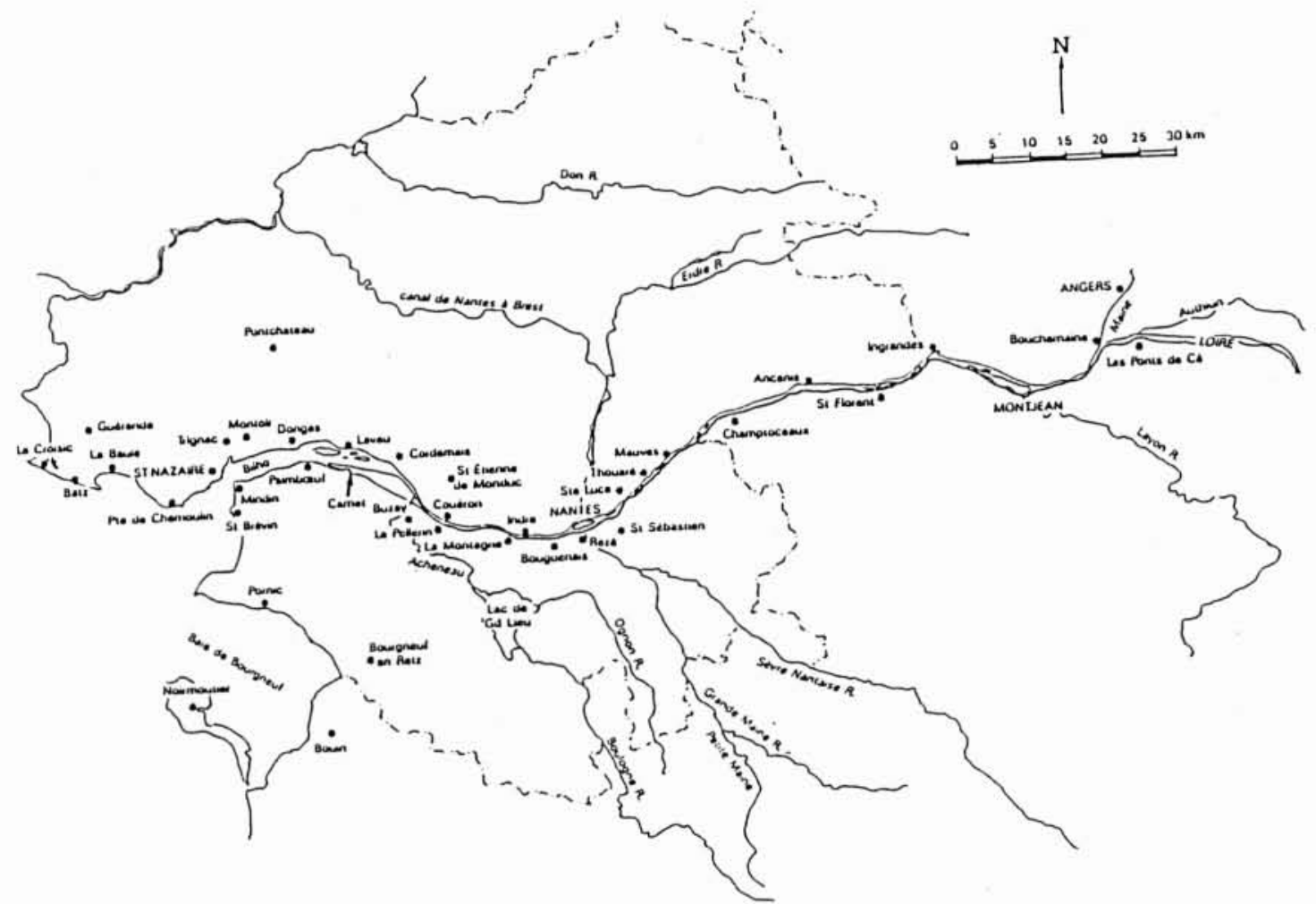

1. Partie aval du bassin de la Loire.

\section{Introduction}

Depuis plusieurs années, à chaque période estivale, on observe dans l'estuaire de la Loire (fig. l) une mortalité importante de mulets blancs (Liza ramada) adultes. Sans négliger évidemment l'éventualité d'une mort naturelle, la plupart des scientifiques s'accordent cependant pour attribuer cette mortalité à un déficit sévère en oxygène dissous des eaux de l'estuaire. Par ailleurs, le Comité scientifique pour l'environnement de l'estuaire de la Loire (CSEEL) [3] signale le rôle d'obstacle que peut jouer un déficit en $\mathrm{OD}\left({ }^{* * *}\right)$ important quant à la pénétration de la crevette grise et d'autres organismes migrateurs dans l'estuaire de la Loire.

Les organismes chargés de l'environnement de l'estuaire souhaitent disposer d'un outil de gestion permettant d'évaluer l'impact des apports fluviaux (Loire amont) et des rejets directs de l'agglomération nantaise afin de comprendre les origines de ce déficit.
La complexité du problème et le travail considérable à réaliser pour la mise en place d'un modèle mathématique reproduisant la distribution et l'évolution de l'OD dans l'estuaire, nous oblige à procéder en plusieurs étapes. Dans cet article, nous présenterons les connaissances acquises dernièrement sur le problème de I'OD dans l'estuaire. La réalisation pratique ainsi que les applications du modèle proprement dits feront l'objet d'une communication ultérieure.

(*) IFREMER, Service Environnement Méditerranéen, B.P. 330,83507 La Seyne-sur-Mer.

(**) Port Autonome de Nantes/St-Nazaire, Service Etudes des Eaux, B.P. 1052, 44037 Nantes Cedex.

$(* * *)$ Le déficit en oxygène dissous (DOD) correspond à la différence entre la valeur de l'OD à saturation et la valeur en OD mesurée ; une manque d'OD se traduit par des valeurs positives, une sursaturation par des valeurs négatives. 


\section{Effect of maximum tunbidity zone on the variations of dissolved oxygen concentrations : example of the estuary of the Loire}

The dissolved oxygen is a sensitive parameter for the environment quality. It is subjected to important variations in macrotidal estuaries with significant results on the fauna. It's why we can notice the important mullet death note in the Estuary of the Loire during sommer time. The supply of biodegradable organic substances in the maximum turbidity zone led to an important dissolved oxygen demand. It's the example of the Estuary of the Loire where we can watch this main fonction. A detailed study has begun on the dissolved oxygen cycle to compare the biochemical particulate and dissolved oxygen demand. This study collects in situ parameters, either of the environment evolution or of the dissolved oxygen cycle with specific researches on the rate of degradation of the particulate and dissolved organic substances.

\section{Distribution et évolution de l'oxygène dissous dans l'estuaire de la Loire}

Le gradient spatial de l'oxygène dissous: De nombreux auteurs ont étudié la distribution de l'OD dans l'estuaire de la Loire (Université de nantes) [2], Guillaud [5], SALIOT et al. [9]. Ils retrouvent généralement une distribution caractéristique amont-aval (profil de courbe en sac) avec un minimum de $1 \mathrm{mg} / \mathrm{l}$ situé en aval de Nantes et correspondant à l'emplacement du bouchon vaseux (fig. 2 et 3). Ce phénomène a déjà été signalé par MORRIS et al. [6] sur l'estuaire de la Tamar en Grande-Bretagne.

La valeur du minimum en OD dépend d'un ensemble de facteurs parmi lesquels les plus importants sont la température de l'eau, la variation des débits fluviaux, le coefficient de marée et la quantité de MES au sein du bouchon vaseux. De part et d'autre du bouchon vaseux, et plus particulièrement en amont de l'estuaire, les teneurs en OD trouvées sont fortes en raison surtout d'une production algale importante (sursaturation $125 \%$ ).

L'évolution temporelle de l'oxygène dissous: Depuis 1985, le Port Autonome de Nantes-St-Nazaire a installé au Pellerin (PK 38), sur le bord de l'estuaire et en surface, un appareil (MOAMED) destiné à enregistrer en continu l'évolution en fonction du temps des paramètres suivants : $\mathrm{T}^{\circ}$, conductivité (salinité), Ph, OD (fig. 4). Le cycle de la marée $(12 \mathrm{~h} 25)$ est visible au niveau de chacun des paramètres. Pour l'OD et le $\mathrm{pH}$, on observe un cycle lunaire, fonction du coefficient de marée et un cycle de marée à deux maxima et à deux minima.

Malgré des vitesses de courant faibles en mortes eaux entraînant une réaération des eaux moins efficace, on observe des niveaux d'oxygène plus forts qu'en vives eaux.

L'explication est à trouver au niveau des matières en suspension et plus particulièrement de leur composante organique biodégradable :

- en vives eaux, la plupart du matériel particulaire est en suspension: il créé une demande importante en OD qui s'ajoute à celle du matériel dissous ;

- en mortes eaux, ce matériel organique se dépose au fond (crème de vase) transformant une DBO particulaire en suspension (demande par unité de volume) en une demande benthique (par unité de surface), quantitativement plus faible. Une réoxygénation progressive du milieu peut se produire par réaération et par redémarrage de la production primaire locale, induite par une augmentation de la pénétration lumineuse (due au dépôt des MES).

Reconstitution du gradient amont-aval à partir de son évolution temporelle en un point fixe de l'estuaire: Nous avons repris des périodes courtes d'enregistrement, de l'ordre de la journée, afin de mettre en évidence l'influence du cycle de marée sur l'évolution de l'OD. La figure 5 présente l'évolution de la salinité et du DOD (au Pellerin PK 38) au cours des journées des 9 et 10 août 1986 (coef. 85-83, $Q=192 \mathrm{~m}^{3} / \mathrm{s}$ ). A chaque maximum ou minimum de salinité (respectivement valeurs aval et amont) correspond un minimum en $O D$, ce qui montre que nous nous trouvons dans la zone du maximum de turbidité et du maximum de DOD, celui-ci "défilant " devant l'enregistreur avec une diminution des valeurs vers l'aval ou vers l'amont. Nous avons porté sur la figure 6 une reconstitution amont-aval de ces gradients, en restituant le long de l'estuaire les positions respectives des différentes masses d'eau au moment de l'étale de pleine mer locale du courant. On peut observer que la valeur maximale du DOD à ce moment-là $(7,75 \mathrm{mg} / \mathrm{l})$ correspond à une salinité de $3 \%$. Par ailleurs, à l'aide des diagrammes $\mathrm{OD} /$ salinité, nous avons mis en évidence une légère réoxygénation des eaux de surface (inférieure à $0,2 \mathrm{mg} / \mathrm{l}$ ) au moment des étales.

La figure 7 montre les enregistrements correspondant à deux appareils situés l'un au Pellerin, l'autre au Carnet du 4 et 21 août 1986. La section mouillée de l'estuaire aval de la Loire suit une évolution exponentielle en fonction de la distance par rapport à l'embouchure. Ceci entraîne une distribution aval-amont de la salinité en double exponentielle (Romana [7]). La transformation logarithmique de la salinité permet donc de visualiser les gradients des autres paramètres en fonction d'une échelle plus conforme à une distance réelle sur le trajet amont-aval. Les maxima en DOD correspondent également à des salinités comprises entre 1,5 et $3 \%$. 

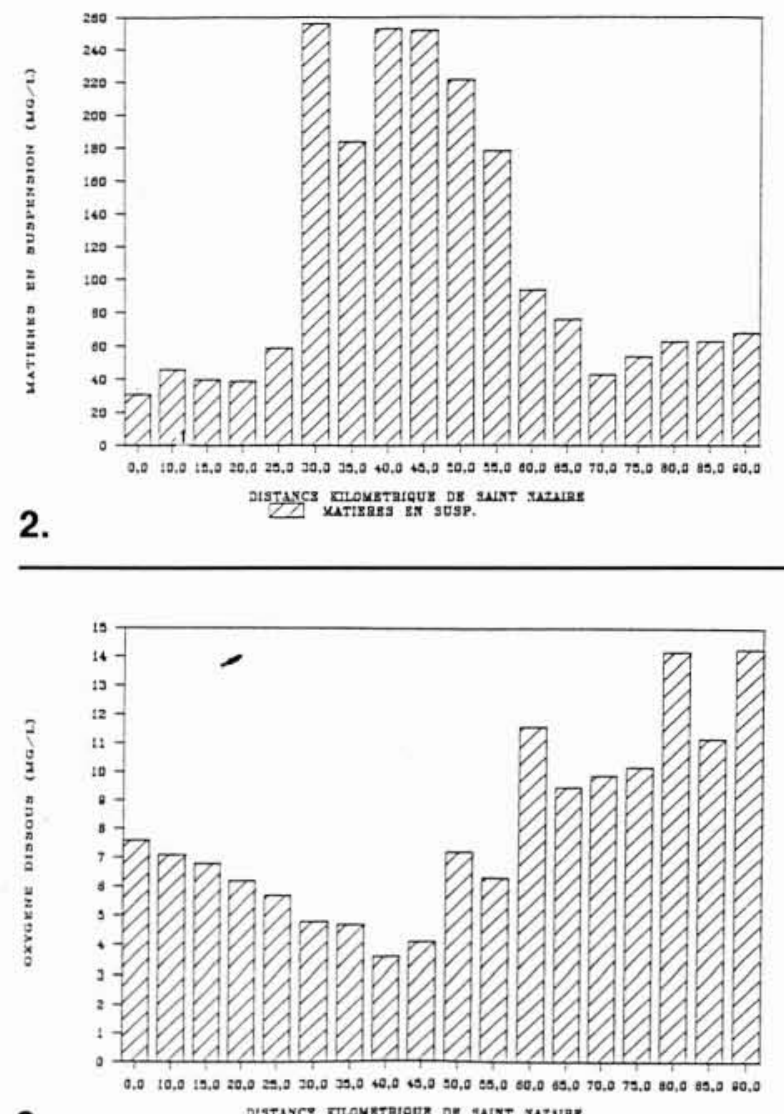

3.

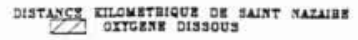

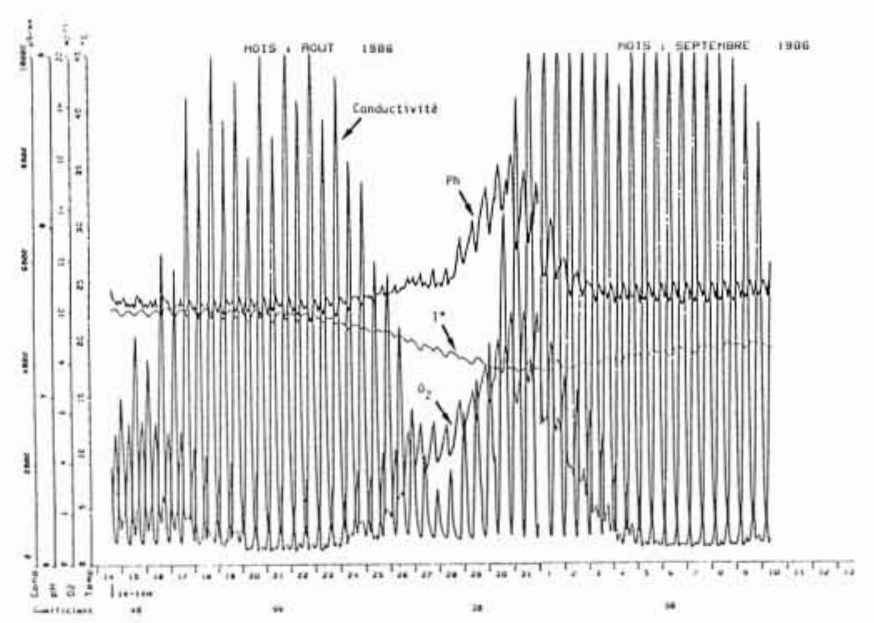

4.

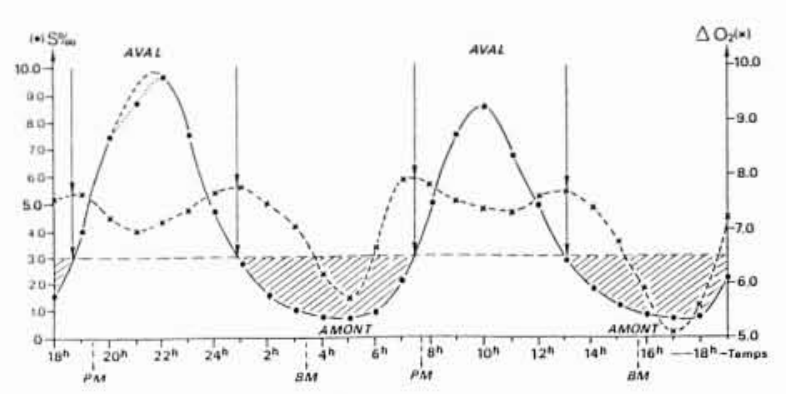

5.
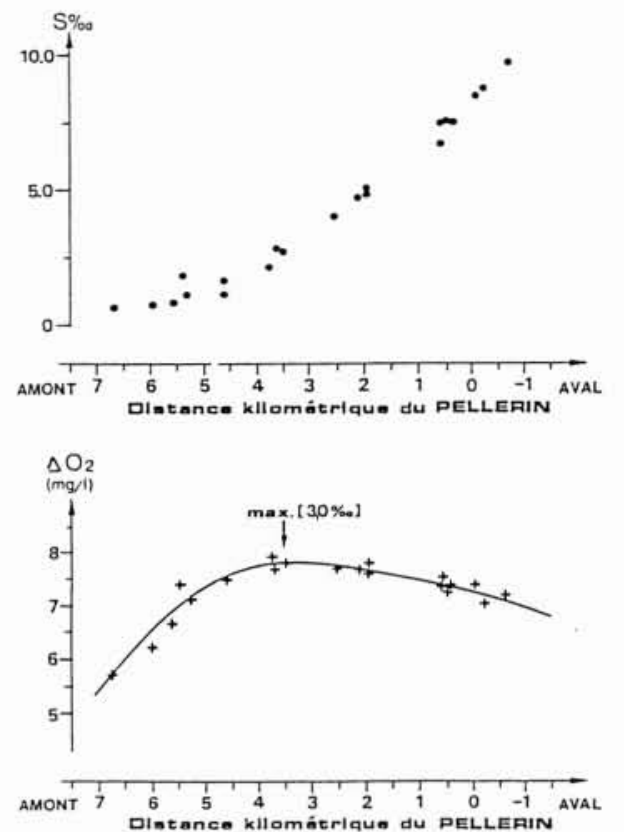

6.
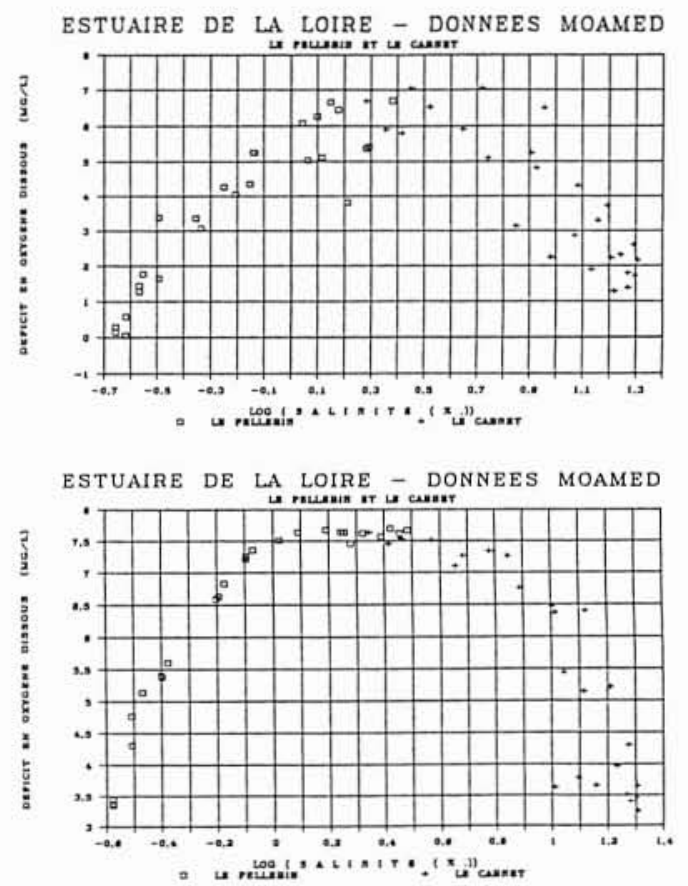

7.

2. Distribution longitudinale des matières en suspension. Campagne du 27 août 1988.

3. Distribution longitudinale de loxygène dissous. Campagne du 27 aoùt 1988.

4. Evolution des paramètres. Site: Le Pellerin.

5. Evolution au cours d'une journée de la salinité et du déficit en oxygène dissous au Pellerin.

6. Distribution de la salinité et du déficit en oxygène dissous en fonction de la distance.

7. Evolution du déficit en oxygène dissous en fonction de la salinité. Enregistrements du 4 et du 21 août 1986 effectués par le Mohamed du Pellerin et du Carnet. 


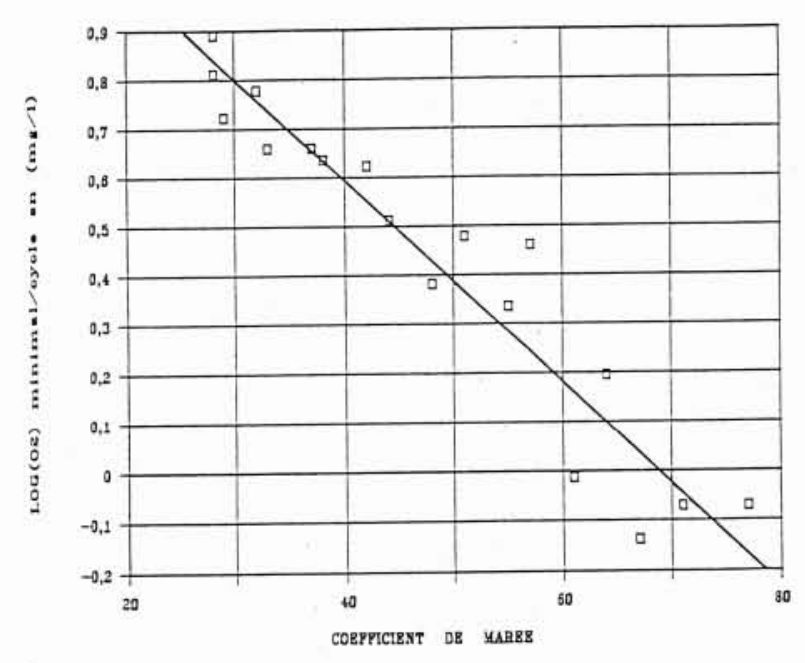

8. Corrélation entre la valeur minimale d'oxygène dissous observée sur une marée au Pellerin (PK 38) en fonction du coefficient de marée. Période du 24 août au 4 septembre 1986.

Relation entre le minimum de l'oxygène dissous et le coefficient de marée : L'existence d'un cycle lunaire et d'un cycle de marée permettant de retrouver la valeur maximale du DOD dans l'estuaire laisse supposer la possibilité de relier cette valeur maximale au coefficient de marée par une loi empirique. Nous avons porté sur la figure 8 le logarithme de la valeur minimale en OD trouvée par cycle de marée, en fonction du coefficient de marée entre le 24 août et le 4 septembre. Le maximum d'OD se présente une journée plus tard vis-à-vis du coefficient de marée minimal. La corrélation porte donc sur des couples de valeurs qui ont été décalés d'une journée. A partir d'un échantillon de 19 valeurs, la loi obtenue est la suivante (coef. de régression $=0,96)$ :

$\log ($ ODmin. $)=1,2343-0,0136$ (coef. marée)

Cette loi met en évidence 3 faits majeurs :

- en plein été, c'est-à-dire en période chaude et en étiage, le minimum estuarien en OD peut varier énormément ;

- le maximum en OD atteint en mortes eaux est proche de la saturation : ceci indique que sans bouchon vaseux, il n'y aurait aucun problème d'OD dans l'estuaire, et ce dans les mêmes conditions de débit et de tempétature;

- l'anoxie serait atteinte pour des coefficients de marée supérieurs à 80 . Cette valeur est aussi la limite à partir de laquelle toute la crème de vase est érodée. Cette situation est atteinte pour $11 \%$ des marées en période estivale.

\section{Le cycle de l'oxygène dissous dans l'estuaire de la Loire: Approche de la modélisation et quantifica- tion des mécanismes}

La réalisation d'un modèle mathématique réaliste de l'évolution de l'OD dans l'estuaire de la Loire, susceptible de répondre aux soucis des aménageurs, passe par une analyse détaillée des différents mécanismes impliqués. Nous citerons brièvement les modèles développés pour résoudre le transport, la diffusion et l'évolution des matières en suspension. Nous développerons plutôt les premières études réalisées sur la demande biochimique en oxygène, en particulier sur les cinétiques de consommation d'oxygène par la dégradation de la matière organique dissoute et particulaire pélagique.

Les phénomènes hydrodynamiques et hydrosédimentaires: Un modèle unidimensionnel a été développé entre St-Nazaire et Ancenis, permettant d'évaluer le transport et le mélange de l'oxygène le long de l'estuaire. Les phénomènes hydrosédimentaires (décantation, érosion, etc...) agissent indirectement sur l'oxygène dissous en faisant varier la concentration en matières en suspension et par ce biais la concentration en DBO particulaire et la production primaire.

Les variations de MES peuvent être importantes à des échelles de temps aussi différentes que celles du cycle de marée, du cycle lunaire ou du cycle saisonnier. Leur distribution le long de l'estuaire et leur évolution au cours du temps peut être reproduite soit par l'intermédiaire des mesures réalisées par différentes conditions de débit et de coefficient de marée, soit à l'aide d'un modèle hydrosédimentaire. Un modèle mathématique hydrosédimentaire est en cours de développement qui tentera de reproduire la formation et l'évolution de bouchon vaseux sur un cycle annuel (P. LE HIR).

La demande biochimique en oxygène : Elle agit différemment selon que la matière organique biodégradable correspondante se présente sous forme dissoute ou particulaire. D'autre part, son devenir dans l'estuaire n'est pas le même suivant sa présentation.

Mesure de la DBO en 4 points par respirométrie: Les DBO dissoutes et particulaires ont été mesurées le 26 août en 4 stations choisies de façon à disposer d'échantillons ayant les caractéristiques suivantes:

A) Donges : forte salinité et faible MES (aval de l'estuaire).

C) Sardine: moyenne salinité et forte MES (aval du bouchon).

D) Cheviré: salinité nulle et forte MES (amont du bouchon).

B) Mauves : salinité nulle et faible MES (amont de l'estuaire).

Les mesures ont été réalisées à l'aide d'un respiromètre SAPROMAT qui permet d'évaluer la consommation d'oxygène en fonction du temps pour des périodes longues (ici 1 mois) tout en gardant constantes les conditions du milieu. La température des échantillons a été maintenue à $20^{\circ} \mathrm{C}$, température de l'eau de l'estuaire au moment du prélèvement.

Ces quatre prélèvements ont fait l'objet de 3 types de sous-échantillons :

- l'échantillon «brut»;

- un échantillon "filtré " par centrifugation donnant la DBO dissoute ; 


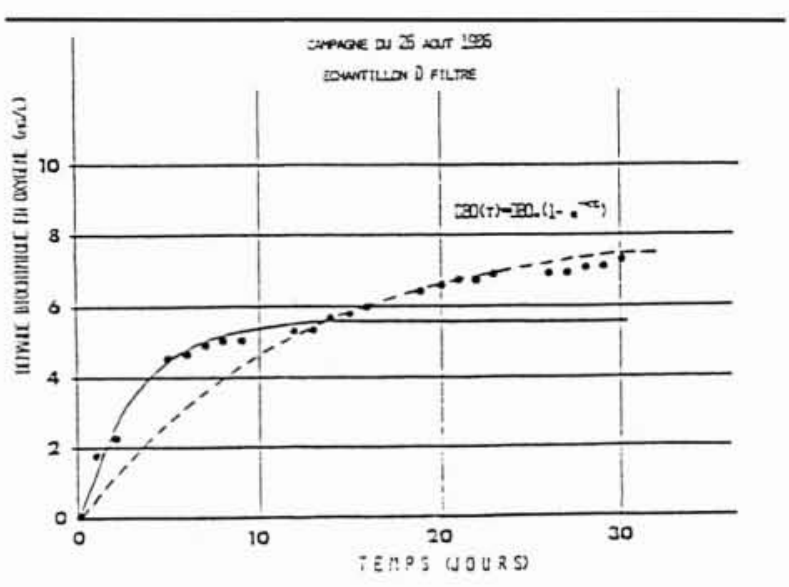

9. Ajustement de lévolution de la DBO en fonction du temps par deux cinétiques de dégradation.

Tableau 1. Demande biochimique ultime en OD (mg/l) pour la première asymptote exponentielle.

\begin{tabular}{|c|c|c|c|c|c|c|}
\hline $\mathrm{s}^{\circ}$ & ECHANTILLON & $2 \pi$ & BRUT & FILTRE & RECONST. & RECONST./BRUT \\
\hline A & DONGES & 11.5 & 3.03 & 4.46 & 2,16 & $33 *$ \\
\hline c & SARDINE & 31.5 & 12,80 & 4.93 & 3.66 & $43 *$ \\
\hline$D$ & CHEVIRE & 50.7 & 11.66 & 5.60 & 6.00 & $52 *$ \\
\hline B & GAUVES & 72.0 & 13,88 & 9.31 & 3.30 & $26 x$ \\
\hline
\end{tabular}

Tableau 2. Demande biochimique ultime en OD (mg/l) pour le deuxième asymptote exponentielle.

\begin{tabular}{ll|rrrrr}
\multicolumn{2}{c|}{ M E EHANTTLLON $^{\text {PR }}$} & BRUT & FTLTRE & RECONST. RECONST./BRUT \\
\hline A & DONGES & 11.5 & 4.62 & 6.09 & 4.41 & $42 *$ \\
C & SARDINE & 31.5 & 12.90 & 6.34 & 6.73 & $50 *$ \\
D & CHEVIRE & 50.7 & 13.77 & 8.10 & 9.38 & $54 *$ \\
B & MAUVES & 72.0 & - & 14.36 & 5.08 & $26 *$
\end{tabular}

Tableau 3. Cinétique de dégradation de la DBO obtenues sur des échantillons prélevés en estuaire de la Loire le 26 août 1986, et analysé par le SAPROMAT. Les valeurs $k 1$ et $k 2$, exprimées en $j-1$, représentent les cinétiques de la première et deuxième exponentielle respectivement.

\begin{tabular}{|c|c|c|c|c|}
\hline ECHANTILLON & $\mathrm{PR}$ & TYPE & $\mathrm{K} 1$ & $\mathrm{~K} 2$ \\
\hline \multirow{3}{*}{$\begin{array}{c}\hat{A} \\
\text { DONGES } \\
\text { S\&. }: 20,6 \\
\text { MES }: 53,9\end{array}$} & \multirow{3}{*}{11.5} & BRUT & 0.305 & 0,085 \\
\hline & & FILTRE & 0.242 & 0.083 \\
\hline & & RECONSTITUE & 0.159 & 0.071 \\
\hline \multirow{3}{*}{$\begin{array}{c}C \\
\text { SARDINE } \\
\text { S } \$:: 6.2 \\
\text { MES }: 217.7\end{array}$} & \multirow{3}{*}{31,5} & BRUT & 0.096 & 0.079 \\
\hline & & FILTRE & 0,324 & 0.074 \\
\hline & & RECONSTITUE & 0,129 & 0,051 \\
\hline \multirow{3}{*}{$\begin{array}{c}\text { D } \\
\text { CHEVIRE } \\
\text { S \$. : } 0.1 \\
\text { MES }: 180.9\end{array}$} & \multirow{3}{*}{50.7} & BRUT & 0.131 & 0,096 \\
\hline & & FILTRE & 0.324 & 0.085 \\
\hline & & RECONSTITUE & 0.102 & 0,047 \\
\hline \multirow{3}{*}{ 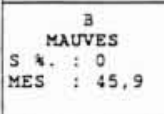 } & \multirow{3}{*}{72.0} & BRUT & 0.230 & - \\
\hline & & FILTRE & 0.240 & 0.085 \\
\hline & & RECONSTITUE & 0.139 & 0.085 \\
\hline
\end{tabular}

- un échantillon « reconstitué » donnant la DBO particulaire préparé de la façon suivante : le culot résultant de la centrifugation est remis en suspension dans un mélange d'eau douce et d'eau de mer méditerranéenne (pauvre en matières organiques) dont la salinité résultante est celle de l'eau dont le culot est issu.

Les courges d'évolution de la DBO en fonction du temps semblent être des fonctions exponentielles dont la formule est donnée par :

$$
\text { DBO }(t)=\mathrm{DBO} u \cdot\left(1-e^{-k t}\right)
$$

$\mathrm{DBO} u=\mathrm{DBO}$ ultime (dégradation totale de la matière organique)

$\mathrm{k}=$ cinétique de transformation.

Un traitement mathématique permet de retrouver les couples $(\mathrm{DBO} u, k)$ correspondant à 2 fonctions exponentielles asymptotiques qui se font suite. La figure 9 met en parallèle les DBO5 mesurées et calculées en fonction du temps. Les autres prélèvements donnent tous des courbes qui se décomposent de la même façon. La deuxième exponentielle asymptotique apparaît, pour tous les échantillons, aux environs du $10^{\mathrm{e}}$ jour d'expérimentation. Les DBO ultimes correspondant à la première et à la deuxième exponentielle négative sont présentées sur les tableaux 1 et 2 . Un examen des données portées sur les deux tableaux fait apparaître que :

— mis à part le prélèvement $A$, la somme des DBO ultimes des échantillons "filtrés" et "reconstitués» restitue approximativement la DBO ultime de l'échantillon «brut ";

- la véritable $\mathrm{DBO} u$ particulaire représente entre 40 et $50 \%$ du total dans l'estuaire et $25 \%$ à l'amont (ces mesures ont été faites en périodes de mortes eaux);

- la DBOu dissoute décroît l'amont en aval ce qui s'interprète comme un appauvrissement progressif de la DBO du fait de sa dégradation le long de l'estuaire et de sa dilution dans l'eau de mer pauvre en DBO :

- les $\mathrm{DBO} u$ particulaires présentent des valeurs maximales au niveau du bouchon vaseux ce qui confirme l'importance de celui-ci vis-à-vis de la demande en oxygène.

La présence de 2 droites correspondant à 2 cinétiques peut être expliquée par la présence de deux stocks différents de matière organique biodégradable, l'un « ancien " à cinétique de dégradation lente, l'autre comportant de la matière organique "fraîche ", à cinétique de dégradation plus rapide.

Analyse des cinétiques de dégradation: Il n'existe dans l'estuaire de la Loire aucune information concernant les cinétiques de dégradation de la DBO. La campagne de mesures réalisée le 26 août 1986 nous a permis, à l'aide du SAPROMAT, d'obtenir des informations fiables sur les vitesses de dégradation du matériel organique total, filtré et particulaire, à différents endroits de l'estuaire. Les résultats des cinétiques (coefficient $k$ dans l'éq. (3) sont présentés dans le tableau 3.

Les cinétiques de dégradation à long terme: Les cinétiques les plus faibles correspondent aux échantillons particulaires prélevés dans le bouchon vaseux. La moyenne est 
de $0,049 \mathrm{j}-1$. Les échantillons filtrés présentent des cinétiques remarquablement constantes $(0,085)$.

Les cinétiques de dégradation à court terme : Les cinétiques de l'échantillon brut sont plus faibles dans le bouchon vaseux $(0,0096-0,131)$ qu'à l'extérieur $(0,305-$ 0,230 ). Par contre, pour l'échantillon filtré, c'est le contraire : 0,324 pour le bouchon, 0,240 à l'extérieur de celui-ci. Ces dernières sont les cinétiques les plus fortes rencontrées.

Les échantillons particulaires ont les cinétiques les plus lentes. Les échantillons bruts, prélevés dans le bouchon vaseux, ont des cinétiques très proches de celles des échantillons particulaires, du fait de leur quantité importante en MES qui rend la DBO particulaire prédominante. Ces valeurs indiquent bien l'ancienneté du matériel organique particulaire du bouchon vaseux.

Comparaison avec les données des autres estuaires européens : D'autres études sur le cycle de l'OD dans les estuaires (WEEB [11], ANONYMous [2] et VAN Es [10]) utilisent deux et parfois trois compartiments de carbone organique caractérisés par des cinétiques de dégradation différentes. Nous avons porté sur le tableau 4 les valeurs trouvées par ces différents auteurs en Hollande et en Grande-Bretagne, et nous les comparons à celles trouvées sur la Loire pour les échantillons bruts.

Les valeurs de $k 2$ (cinétique lente) sont tout à fait comparables à celles trouvées par les autres auteurs. Les estuaires étudiés par ces derniers auteurs présentent des bouchons vaseux à teneurs en MES plus faibles, qui sont donc difficilement comparables aux estuaires français pour les cinétiques rapides. $\mathrm{Si}$ l'on compte maintenant les valeurs amont et aval de $k 1$ pour la Loire, la moyenne est alors beaucoup plus proche de celle obtenue par les autres auteurs : $0,268 \mathrm{j}-1$.
Tableau 4. Cinétiques de dégradation de la matière organique dans les trois catégories prises en compte par les différents auteurs.

\begin{tabular}{|l|c|c|c|}
\hline $\boldsymbol{A}$ U T E U R & $\mathrm{K} 1$ & $\mathrm{~K} 2$ & $\mathrm{K3}$ \\
\hline HEBB (9) & 0,234 & 0,046 & - \\
\hline VAN ES (11) & 0,280 & 0,100 & 0.017 \\
\hline ANONYMOUS (10) & - & 0,088 & 0,018 \\
\hline CETTE ETUDE & 0.190 & 0.087 & - \\
\hline
\end{tabular}

Tableau 5. Présentation des ètudes. Stratégie adoptée pour la mise en place des modèles.

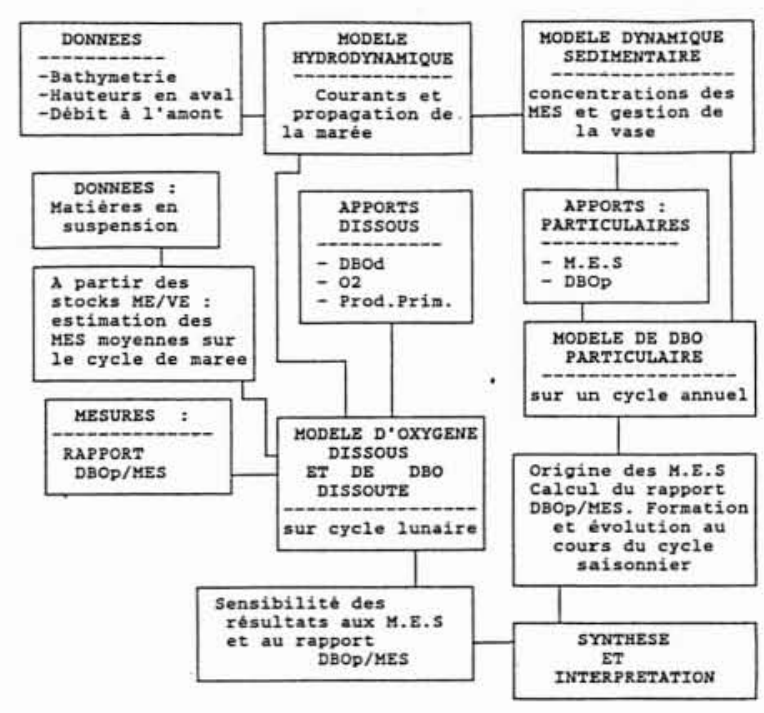

\section{Conclusions}

Ces résultats ont permis de définir les différentes étapes à mettre en œuvre dans le développement d'un modèle visant à reproduire l'évolution de l'oxygène dissous dans l'estuaire de la Loire. Ainsi, on a pu mettre en évidence que les problèmes d'anoxie étaient fortement reliés à la présence du bouchon vaseux. Au-delà d'un coefficient de marée de 80 et pour des températures supérieures à $20^{\circ} \mathrm{C}$ (périodes estivales et post-estivales), les conditions anoxiques semblent atteintes quasi-systématiquement. Le comportement physico-chimique de la matière organique est différent selon qu'elle se présente sous forme dissoute ou sous forme particulaire (stockée dans le bouchon vaseux). La démarche suivie consiste ici à séparer le problème en deux étapes :
- en premier lieu, l'oxygène et la DBO dissoute sont modélisés ; la DBO particulaire étant reliée à la distribution moyenne des MES par le rapport DBO5/MES mesuré ;

- en second lieu, la formation et l'évolution du bouchon vaseux et de sa composante biodégradable est reproduite sur un cycle annuel afin de retrouver l'impact respectif des apports amonts de la Loire et des rejets dans l'estuaire.

La mise en place de ces différents modèles (voir tabl. 5) a pour but de déterminer les causes de l'anoxie et de rechercher des solutions pour l'amélioration de la qualité de l'eau dans l'estuaire de la Loire. 


\section{Bibliographie}

[1] ANONYME. - Etude générale d'environnement de l'estuaire de la Loire préalable à son aménagement industrialo-portuaire. Université de Nantes. Parie III : Etude physico-chimique du milieu liquide, 1977.

[2] Anonymous. - Dept. Sci. Ind. Research. Technical paper 11. H.M. Stationaru Office, 1964.

[3] COMITÉ SCIENTIFIQUE POUR L'ENVIRONNEMENT DE L'ESTUAIRE DE LA LOIRE. - Publications du Centre national pour l'exploitation des océans. Rapports scientifiques et techniques, $\mathrm{n}^{\circ} 55$, 1984

[4] Caunet G. - Thèse de Doctorat d'Etat de l'Université de Perpignan, 1985.

[5] Guillaud J.F. - La qualité des eaux dans l'estuaire de la Loire. Rapport provisoire dans le cadre du CSEEL. Août 1983.

[6] Morris A.W., Loring D.H., Bale A.J., Howland R.J.M., Mantoura R.F., Woodward E.M.S. - Oceanologica Acta, 1982, Vol. $5, \mathrm{n}^{\circ} 3$.

[7] Romana L.A. - Estuaire de la Gironde. Campagne "Libellule I" du 26 août 1981. Rapport CNEXO-Environnement. 1982.

[8] Romana L.A. - Nitrification en estuaire de la Seine. Influence des matières en suspension. Rapport IFREMER, 1984.

[9] Saliot A., Lorre A., Marty J.C., Scribe P., TronczynSki J., Meybeck M., Desserey S., Marchand M., Caprais J.C., Cauwet G., Etcheber H., Relexans J.C., Ewald M., Berger P., Belin C., Gouleau D., Billen G., Somville M. - Oceanologica Acta, 1984, Vol. 7, n 2.

[10] Van Es F.B., Ruardis P. - Netherlands Journal of Sea Research, 15 (3/4), 1982.

[11] WEEB A.J. - Un modèle mathématique en oxygène dissous dans l'estuaire du Forth. Communication personnelle, 1987. 